\title{
Uma Estratégia de Alocação da Etapa de Requisitos como Apoio ao Gerenciamento de Projetos em Desenvolvimento Distribuído de Software
}

\author{
Euclides Alfredo Matusse ${ }^{1}$, Edson A. Oliveira Junior ${ }^{1}$, Elisa Hatsue Moriya \\ Huzita $^{1}$, Tania Fatima Calvi Tait ${ }^{1}$ \\ ${ }^{1}$ Departamento de Informática - Universidade Estadual de Maringá (UEM) \\ Caixa Postal 87.020-900 - Maringá - PR - Brazil \\ ematusse27@gmail.com, \{edson, tait, emhuzita\}@din.uem.br
}

\begin{abstract}
Distributed software development (DSD) has been an approach increasingly adopted by companies. Hence, managing the distribution of steps in a development process among distributed teams is quite challenging. Therefore, defining strategies that consider socio technical aspects involving people, technology, and process metrics can provide the necessary support. This paper presents the experimental validation using GQM to evaluate a strategy that considers the estimated effort as an intrinsic element. The results show that these metrics are indicators to allocate requirements step in DSD offshore outsourcing model.
\end{abstract}

Resumo. O desenvolvimento distribuído de software (DDS) tem sido uma abordagem cada vez mais adotada pelas empresas. Logo, gerenciar a distribuição de etapas de um processo de desenvolvimento entre equipes distribuídas é bastante desafiador. Portanto, definir estratégias que consideram aspectos sócio técnicos envolvendo pessoas, tecnologia, processo e métricas podem oferecer o apoio necessário. Este artigo apresenta uma validação experimental, usando GQM, para avaliar uma estratégia que considera a estimativa esforço como elemento intrínseco. Os resultados evidenciam que tais métricas constituem-se em indicadores para alocar requisitos em DDS - modelo offshore outsourcing.

\section{Introdução}

Em Desenvolvimento Distribuido de Software, a especificação de requisitos é um fator significativo na qualidade de software [Damian e Zowghi, 2002] e, para tornar isto possível, dados quantitativos que descrevam a realidade do processo precisam ser obtidos e devidamente analisados. Um dos grandes desafios em DDS é a comunicação que devido à distancia geográfica existente, resulta em aumento no custo para estabelecer a comunicação entre as diferentes equipes envolvidas, podendo até torná-la inadequada. Além disso, restringe a possibilidade de realizar a comunicação informal, que tradicionalmente se estabelece entre as pessoas, permitindo com isto que se ajudem e compartilhem experiências. Esta troca de experiências é de grande importância para que os membros das equipes colaborem e, consequentemente, alcancem as metas estabelecidas [Herbsleb e Moitra, 2001]. Tal colaboração pode ocorrer em atividades no decorrer de todo o processo de desenvolvimento. Nesse contexto métricas de software 
têm se mostrado o fator chave para alocação da etapa de requisitos facilitando a oportunidade de melhorar o processo e o produto [Fenton, 1991]. Estudos experimentais conduzidos em vários locais de desenvolvimento de software relatam problemas causados pela falta de habilidades sociais necessárias [Gotel et al., 2010], alocação de equipes e tarefas geograficamente distribuída [Lamersdorf et al., 2009].

Neste artigo, é apresentada uma estratégia que envolve desde a seleção e definição de métricas até a análise dos resultados obtidos, com objetivo de estudar a combinação de métricas da distancia geográfica, temporal [O’Leary e Cummings, 2007] e a estimativa de esforço para realizar a especificação de requisitos (EReq) em DDS [Karner, 1993] medindo-as e por relações (correlações), por forma, a extrair um direcionador (indicador). Os valores observados foram aplicados aos testes de normalidade de Shapiro e Wilk (1956) e Kolmogorov-Smirnov [Corder e Foreman, 2009]. Essas métricas estão relacionadas com fatores técnicos, não técnicos[Ribu., 2001], visando apoiar a gerência de projetos na tomada de decisões baseada em indicadores, contribuindo para a pesquisa e prática da melhoria no processo em DDS. Os resultados foram avaliados com base nos dados fornecidos pelas equipes selecionadas para o experimento e pela respectiva métrica. Este artigo está organizado da seguinte forma: Seção 2 descreve a metodologia adotada na investigação com o método Goal Question Metric (GQM), Seção 3 apresenta os elementos e a estratégia para alocar a EReq em DDS, Seção 4 define as métricas de distancia geográfica, temporal e a estimativa de esforço da equipe em realizar a EReq a ser avaliada e ilustra como a capturá-las, Seção 5 apresenta como o estudo experimental foi planejado e realizado para avaliar as métricas de estimativa de esforço, Seção 6 descreve a fundamentação e analise dos elementos da estratégia e discute os resultados obtidos no presente estudo, e Seção 7 fornece as conclusões e trabalhos futuros.

\section{Metodologia de Pesquisa}

A abordagem GQM (Basili e Rombach,1988) tem como características: a definição de metas (goals): define objetivos relevantes para organização; perguntas (questions): gera um conjunto de perguntas que define os objetivos mediante aspectos qualitativos, de forma que elas possam ser medidas; e métricas (metrics): especifica um conjunto de métricas que precisam ser coletadas para responder as perguntas geradas. Seguindo a abordagem GQM, foram, inicialmente, estabelecidos os objetivos a serem alcançados na validação experimental considerando as métricas de estimativa de esforço e a estimativa de esforço associado pelos participantes do estudo experimental, visto que a métrica distancia geográfica e temporal foram validadas no trabalho de O’Leary e Cummings, (2007). A Figura 1 descreve os passos da metodologia de pesquisa.

- Passo Definir o alinhamento estratégico, após descrever o problema ou fenômeno de interesse, envolve a definição de questões gerenciais e técnicas a serem respondidas com relação às métricas através do método GQM.

- Passo Definir os objetivos estabelece exatamente quais são as dimensões das metas e objetivos a serem medidos, perguntas e hipóteses para resolver o problema da alocação da etapa de requisitos baseada na correlação das métricas.

- Passo Elaborar conjunto de perguntas que define objetivos. Envolve a elaboração, o refinamento das perguntas e hipóteses que definem os objetivos 
mediante os aspectos qualitativos, de forma que elas possam ser medidas pelos gerentes de projetos.

- Passo Identificar métricas, após terem sido identificados o conjunto de perguntas que, se respondidas, é necessário definir e caracterizar cada elemento das métricas que irão compor a estratégia, a fim de tornar tais medidas aplicáveis.

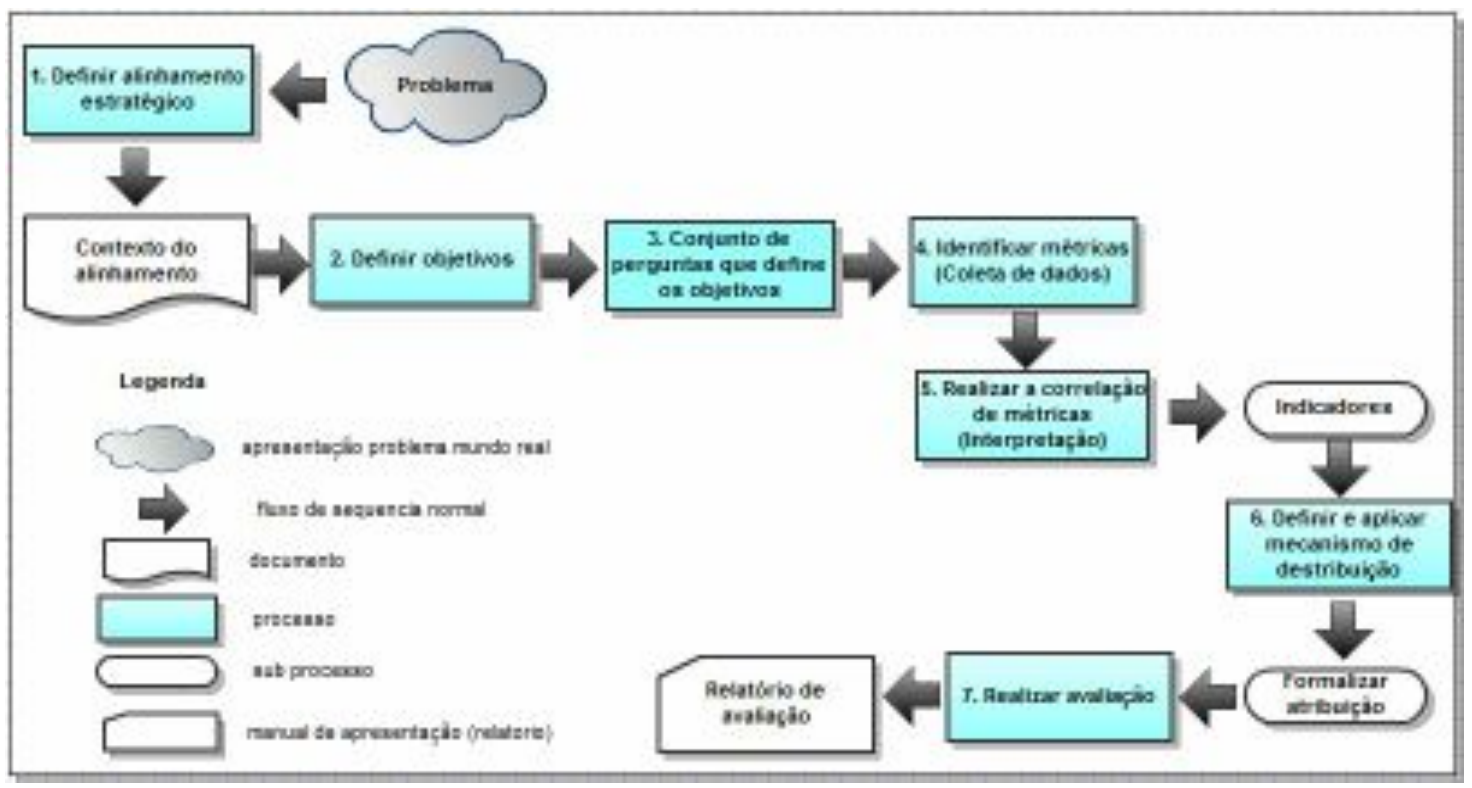

Figura 1. Metodologia da Estratégia

- Passo Estabelecer a correlação de métricas, esta atividade tem como objetivo verificar e analisar a correlação das métricas através de um estudo experimental, descrito na seção 4, visando proporcionar um conjunto de métricas, denominados indicadores capazes de apoiar a gerência de projetos na tomada de decisões na alocação da etapa de requisitos a equipes em várias localidades geográficas.

- Passo estabelecer e aplicar a estratégia de Alocação da Etapa de Requisitos em DDS com base nos requisitos funcionais (RF) e requisitos não funcionais (RNF) do sistema estabelecidos pelo gerente de projetos. Cada uma das descrições dos RF e RNF inclui elementos da métrica estimativa de esforço e níveis de influencia da estimativa de esforço atribuído pela equipe na validação de modo que o seu valor seja aprovado. São também, considerados o valor da métrica da distância temporal e geográfica entre os locais distribuídos.

- Passo Realizar validação, por fim, esta atividade da metodologia compreende a obtenção de um conjunto de informações sobre validação do estudo em relação ao método utilizado no estudo experimental para compreender a natureza dinamica do DDS. Para tanto, é fundamental que sejam registradas anotações ao longo de toda metodologia de pesquisa para garantir a correlação com a finalidade de extrair indicadores. 


\section{EAEReq-DDS: Estratégia de Alocação da Etapa de Requisitos em DDS}

A estratégia tem como objetivo investigar, analisar, adaptar métricas e propor indicadores que são específicos para o DDS para alocar a EReq. Para atingir este objetivo, primeiro foram coletadas evidências sobre métricas para auxiliar a distribuição do processo de software [Matusse et al, 2012]. Esta estratégia abrange 4 grandes áreas de pesquisa, sendo estas: recursos humanos (equipes); métricas; processos e tecnologia. A Figura 2 ilustra os elementos da estratégia. Ao optar por alocar a etapa EReq, além de todas as dificuldades inerentes ao DDS, uma organização começa a enfrentar diversas dificuldades de adaptação tais como; identificação de stakeholders, comunicação, diferenças culturais, rastreamento de requisitos, ambiguidade e falta de clareza que tem que ser levadas em consideração [Prikladnicki e Audy, 2008]. Como pode ser visto na Figura 2, o Workspace (1) provê separação explícita do espaço de trabalho do qual faz parte a equipe distribuída. Através desse espaço a equipe aloca e acompanha os recursos referentes a um projeto, no que tange aos recursos humanos, execução de processos, e tecnologias.

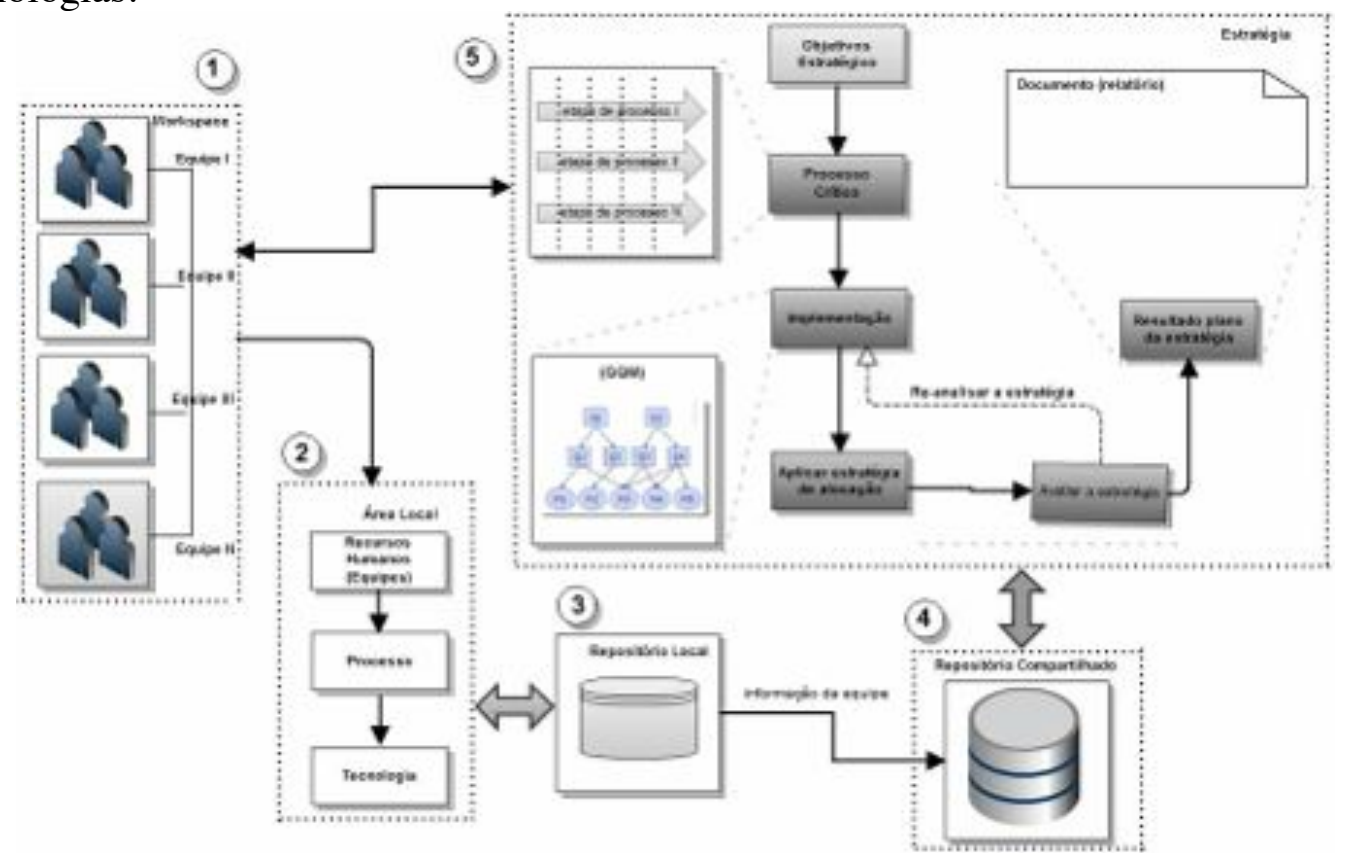

Figura 2. Modelo Conceitual da Estratégia

Assim, a equipe controla suas atividades podendo iniciar, pausar, finalizar ou delegar uma atividade a outro membro da equipe que devem ser encaminhadas a Área Local (2), além de ter acesso às funcionalidades para obter a estratégia. Repositório Local (3), representa o espaço onde são persistidos os dados referentes à Recursos Humanos (Equipes), Processo e Tecnologia. A Área Local contém dados de cada equipe passível de participar de um projeto, os quais serão, posteriormente, enviados para o repositório compartilhado. Repositório Compartilhado (4), responsável por armazenar informações para, através dela, fórmular as metas, objetivos e métricas da estratégia proposta, que será usada para distribuir a EReq em DDS durante o ciclo de vida do projeto. Estrategia (5), abrange os elementos da estratégia proposta. Esses elementos visam a integração das regras de negócio do sistema com implementação do método GQM, de modo a identificar um conjunto de métricas. Por sua vez, essas métricas serão 
correlacionadas para extrair indicadores para distribuir a EReq, como iniciativa para a implementação de melhores práticas para apoiar a gestão de projetos, e gerente de portfólio na tomada de decisões baseada em indicadores. A estratégia permite também avaliar a estratégia e apresentar resultados ao gerente das equipes distribuídas referente à alocação da EReq às equipes participantes do projeto.

\section{Seleção das Métricas para Estudo Experimental}

Para medir a estimativa de esforço envolvido no RF e RNF no desenvolvimento do projeto, são utilizadas tanto as estimativas de tamanho quanto da produtividade da equipe. Foi decidido analisar somente a estimativa de tamanho, uma vez que as outras (prazo e custo) são dependentes dessa. Duas perguntas foram caracterizadas como possíveis de estudar o objeto em questão: (i) "qual é a estimativa de tamanho do RF e RNF especificado para o projeto"? e (ii) "qual é a estimativa de esforço necessário para realizar o RF e RNF do projeto". Assim, para responder a primeira questão utilizou-se a técnica pontos de caso de uso técnico (TUCP) criado por Karner,(1993) por fornecer a estimativa do tamanho logo no início do projeto com base nos modelos de caso de uso construídos. Pretende-se, nessa técnica, utilizar apenas os fatores técnicos e não técnicos [Ribu, 2001] para ser obtida a estimativa de tamanho.

Segundo Karner (1993), a calibragem para a produtividade para cada etapa do ciclo de vida deve ser obtida da base histórica organizacional. Isto porque a experiência da equipe de desenvolvimento pode variar de uma etapa para outra, já que, normalmente, as pessoas envolvidas em uma etapa podem não ser as mesmas de outra etapa. Assim, a estimativa de esforço por etapa do ciclo de vida do projeto é calculado na Eq. 1.

$$
\operatorname{Esforco}_{(\text {etapa })}=\operatorname{TUCP}_{(\text {etapa })} x E F
$$

Onde: Esfoco $_{(\text {etapa })}$ - é a estimativa de esforço por etapa do ciclo de vida, $T U C P_{(\text {etapa })}$ - é a estimativa de tamanho por etapa do ciclo de vida, e $E F$ - corresponde o cálculo de fatores ambientais.

A estimativa de esforço por equipe, definida como segunda questão, é descrita a seguir com a finalidade de extrair a equação Eq. 2.

$$
\text { Esforco }=\frac{\left(\sum \text { Esforco }_{(\text {etapa })}\right)}{\left(N^{2}-N\right) / 2}
$$

Onde: Esforco - é a estimativa de esforço por cada equipe para realizar RF e RNF do projeto, Esforco (etapa) - é a estimativa de esforço por etapa do ciclo de vida, e $N$ - é número total da equipe em todos os locais.

Para medir a métrica da distancia geografia e temporal, duas perguntas foram caracterizadas como possíveis de estudar: (i) "qual é a distancia geográfica das equipes em relação à matriz"?, e (ii) "qual é a precisão da distancia temporal das equipes em relação a matriz"?, afim de atribuir uma regra de negócio identificada na EReq. 
A formulação para a primeira questão consiste em medir a dispersão geográfica (espacial) respeitando as relações de precedências e de recursos [O'Leary e Cummings, 2007], descritas na equação Eq.3.

$$
S D=\sum_{i-j}^{k} \frac{\left(K M_{i j} \times N_{i} \times N_{j}\right)}{\left(N^{2}-N\right) / 2}
$$

Onde: $S D$-é a distancia espacial, $K M_{i j}$ - refere-se ao prazo é a distância entre locais $i$ e $j$ em quilômetros, $N_{i} e N_{j}$ - representam o número de pessoas no local, $k$ - é o número total de locais que modifica em relação a decisão do proprietário do componente de software, $N$ - é o número total de desenvolvedores.

O’Leary e Cummings, (2007), propõem a distribuição temporal para a segunda questão do objetivo que é descrita na equação Eq.4.

$$
T D=\sum_{i-j}^{k} \frac{\left(T Z_{i j} \times N_{i} \times N_{j}\right)}{\left(N^{2}-N\right) / 2}
$$

Onde: $T D$ - é a distancia temporal, $T Z_{i j}$ - refere-se $a$ o prazo é o número de tempo zonas $i$ e $j$ entre localizações, $N_{i} e_{N_{j}}$ - representam do número de pessoas no local, $k$ - é o número total de locais que modifica em relação a decisão do proprietário do componente de software, $N$ - é o número total de desenvolvedores.

\section{Estudo Experimental das Métricas}

Nesta seção, descreve-se o experimento desenvolvido para avaliar empiricamente as métricas propostas como indicadores da alocação da EReq a equipes dispersas em DDS.

\subsection{Planejamento de estudo experimental}

Com base no paradigma GQM proposto por template de Basili et al., (1988), o objetivo do experimento é apresentado como segue:

$\checkmark$ Analisar métricas coletadas da literatura para avaliar a alocação da EReq a equipes dispersas em DDS.

$\checkmark$ Com o propósito de validação.

$\checkmark$ Referente à capacidade de ser usado como indicadores para alocação da EReq de DDS.

$\checkmark$ Do ponto de vista do gerente de projeto.

$\checkmark$ No contexto de alunos de graduação e mestrado da Ciência da Computação da Universidade Estadual de Maringá (DIN-UEM-Brasil), Departamento de Matemática e Informática da Universidade Eduardo Mondlane (DMI-UEMMoçambique), Banco Internacional de Moçambique Millenniumbim (Departamento Funcional de Desenvolvimento) e Portugal.

\subsection{Execução do estudo experimental}

Antes da execução real do estudo, um estudo piloto foi realizado com vista a avaliar a instrumentação do estudo experimental. Para tanto, somente os gerentes locais foram utilizados. Os gerentes utilizaram os mesmos instrumentos do estudo. Os dados obtidos pelo estudo piloto não foram usados para complementar o estudo experimental para 
evitar víeis da informação. Os participantes do estudo, além de frequentarem, pelo menos, curso de graduação na área de computação, estão inseridos no contexto de fábrica de software e possuem conhecimentos mínimos sobre: a Notação UML, mais especificamente sobre modelo de diagramas de casos de uso, classificados por iteração considerando a relevancia do negocio e risco de desenvolvimento; classificação e pesos, guia de classificação e pesos dos fatores ambientais, técnicos e não técnicos que compõe os elementos das métricas e, cenários do estudo, dois modelos de resolução contendo a configuração do estudo experimental por participante. Dezoito pessoas participaram do estudo experimental, sendo cinco graduandos, quatro graduados, oito mestrandos e um mestre. Como foi considerado um nível de amostra pequena, variações com as habilidades dos participantes foram reduzidas. Para o estudo em questão, as seguintes hipóteses foram propostas:

- Hipótese nula $\left(H_{0}\right)$ : não existe correlação significativa entre a métrica estimativa de esforço e a estimativa de esforço da equipe para realizar a EReq associado pelo participante; e

- Hipótese alternativa $\left(H_{1}\right)$ : existe correlação significativa entre a métrica estimativa de esforço e a estimativa de esforço da equipe para realizar a EReq associado pelo participante.

O estudo caracterizou e avaliou a métrica de estimativa de esforço por equipe e a viabilidade de utilizá-las para alocação da EReq a equipes dispersas em DDS. Assim, os mecanismos de análise utilizados foram: análises de estatísticas descritivas acerca das métricas coletadas a partir das configurações geradas, por cada participante, combinadas com análises dos gráficos de dispersão das métricas de estimativa de esforço despendido pela equipe e os níveis de influência da estimativa de esforço associada por participante, e análises de trade-off com o objetivo de priorizar elementos que compõe a métrica para aplicação da estratégia.

\subsection{Análise e interpretação dos resultados do estudo experimental}

$\mathrm{Na}$ validação das métricas foram aplicadas a um conjunto de dezoito configurações distintas geradas por participantes do estudo.
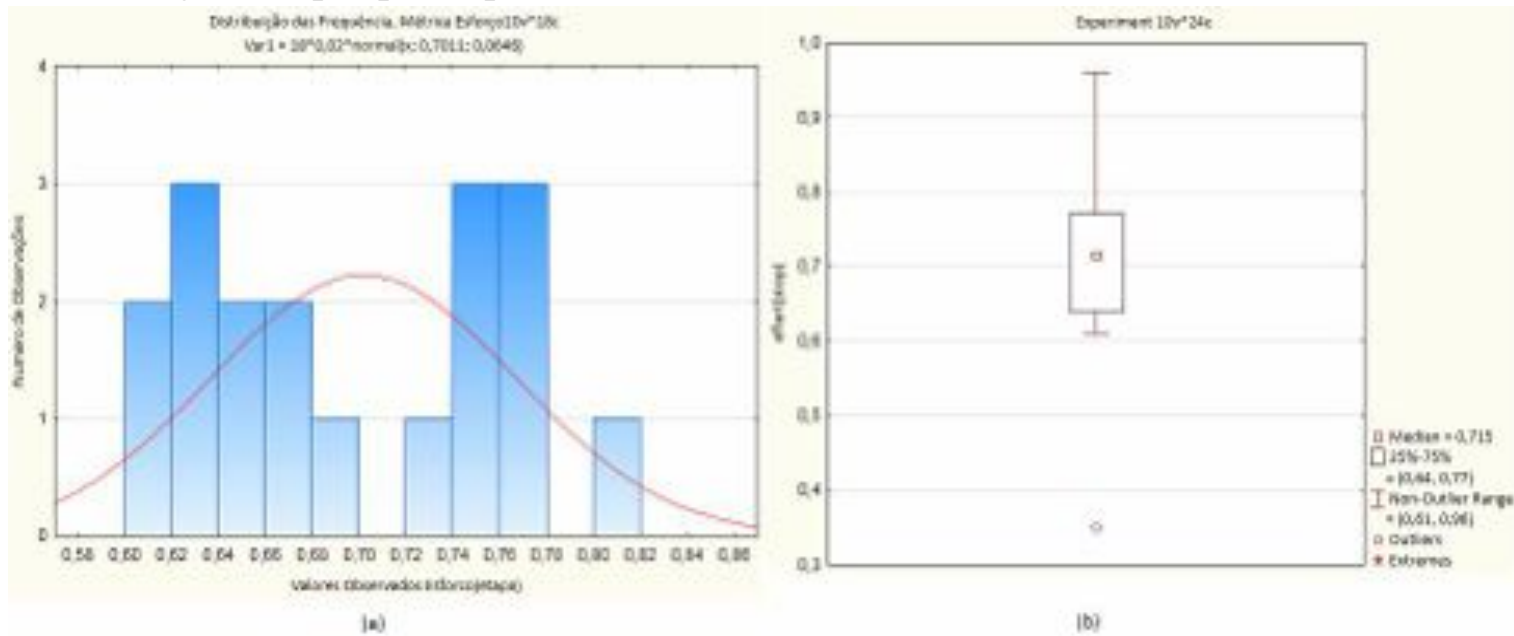

(b)

Figure 3. (a) Distribuição dos valores observados e (b) bloxplot dos valores observados para métrica estimativa de esforço. 
Os valores observados foram aplicados aos testes de normalidade de Shapiro e Wilk (1956) e Kolmogorov-Smirnov [Corder e Foreman, 2009]. Com base na estatística descritiva dos valores da Figura 3(a). A métrica Esforco o valor da média aritmética $(\mu)$ 0,7011 , desvio padrão (ס) 0,0646 e mediana $(\mu d)$ 0,69. A técnica não paramétrica de correlação de Spearman (1904) foi usada para demostrar que a métrica estimativa de esforço por equipe possui uma correlação forte e positiva com o valor de estimativa de esforço associado ao participante. Assim, o resultado obtido forneceu evidências para se rejeitar a hipótese Nula $\left(H_{0}\right)$ e aceitar a hipótese Alternativa $\left(H_{1}\right)$.

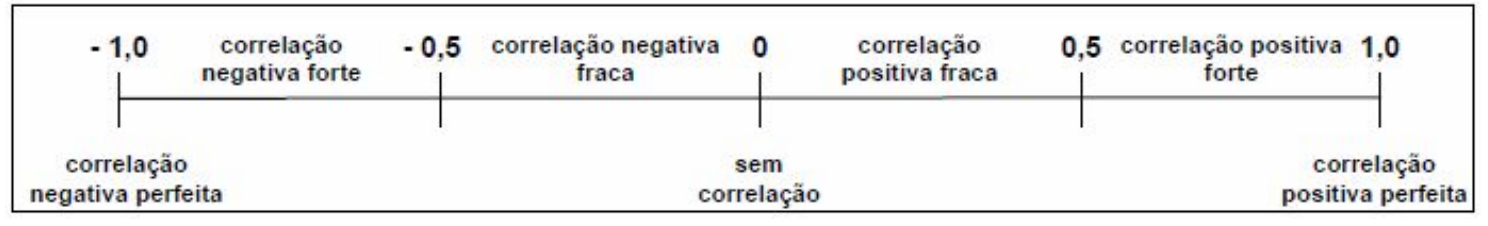

Figure 4. Escala da correlação de Spearman [Spearman, 1904].

A Tabela 1 mostra a correlação de Spearman $\rho($ Corr 1), coeficiente (equação Eq5) é calculado da seguinte forma:

$$
\rho(\text { corr. } 1)=1-\frac{6}{n\left(n^{2}-1\right)} \times \sum d_{i}^{2}
$$

Tal que $n$ é o número de amostra $(N)$

$$
\rho(\text { corr } .1)=1-\frac{6}{18\left(18^{2}-1\right)} \times 336=1-0,38=0,62
$$

A correlação de esforço possui um valor limite de $(0,62)$. Assim, ficou evidenciado que tais métricas são fortemente correlacionadas. Logo, torna-se necessário aplicar métricas propostas em ambientes organizacionais de software para que seja possível reduzir as ameaças externas e obter evidências reais de que elas podem ser utilizadas como indicadores de alocação da EReq em DDS.

Tabela 1. Correlação de Spearman $\rho($ Corr 1$)$ : (Esforco) e (Esforco.part)

\begin{tabular}{|c|c|c|c|c|c|c|}
\hline ID\# & Esforco & $r a$ & Esforco.part & $r b$ & $\begin{array}{c}d \\
|r a-r b|\end{array}$ & $d^{2}$ \\
\hline 1 & 0,77 & 13 & 0,75 & 8 & 5 & 25 \\
\hline 2 & 0,63 & 3 & 0,5 & 1 & 2 & 4 \\
\hline 3 & 0,75 & 9 & 0,75 & 8 & 1 & 1 \\
\hline 4 & 0,77 & 13 & 0,75 & 8 & 5 & 25 \\
\hline 5 & 0,65 & 6 & 0,5 & 1 & 5 & 25 \\
\hline 6 & 0,77 & 13 & 0,75 & 8 & 5 & 25 \\
\hline 7 & 0,64 & 4 & 0,5 & 1 & 3 & 9 \\
\hline 8 & 0,76 & 11 & 0,75 & 8 & 3 & 9 \\
\hline 9 & 0,68 & 7 & 0,5 & 1 & 6 & 36 \\
\hline
\end{tabular}

\begin{tabular}{|c|c|c|c|c|c|c|}
\hline ID\# & Esforco & $r a$ & Esforco.part & $r b$ & $\begin{array}{c}d \\
\text { I ra-rb }\end{array}$ & $d^{2}$ \\
\hline 10 & 0,75 & 9 & 0,75 & 8 & 1 & 1 \\
\hline 11 & 0,61 & 1 & 0,5 & 1 & 0 & 0 \\
\hline 12 & 0,82 & 17 & 0,75 & 8 & 9 & 81 \\
\hline 13 & 0,64 & 4 & 0,5 & 1 & 3 & 9 \\
\hline 14 & 0,76 & 11 & 0,75 & 8 & 3 & 9 \\
\hline 14 & 0,77 & 13 & 0,75 & 8 & 5 & 25 \\
\hline 16 & 0,73 & 8 & 0,75 & 8 & 0 & 0 \\
\hline 17 & 0,82 & 17 & 0,75 & 8 & 9 & 81 \\
\hline 18 & 0,61 & 1 & 0,5 & 1 & 0 & 0 \\
\hline
\end{tabular}




\section{Fundamentação e análise dos elementos da estratégia}

Para alocação da EReq serão levados em consideração os seguintes elementos: i) a correlação $\rho$ (Corr1) descrita na equação Eq5; ii) o tamanho da equipe; iii) os níveis de influência da estimativa de esforço; $i v$ ) a estimativa de esforço despendido pela equipe; e a $v$ ) distancia geográfica e temporal. Portanto, para o efeito de análise de dados nessa fase, torna-se necessário o agrupamento das equipes resultante da estrutura intrínseca da estratégia apresentada na Figura 2. A seguir, são apresentados os elementos descritos para o efeito da alocação da EReq em DDS.

$\checkmark$ Correlação $\rho(\operatorname{Corr} 1)=0,62$ das métricas Esforco e Esforco.part

$\checkmark$ Tamanho da equipe. Este estudo empírico foi realizado com três equipes dispersas em nível global:

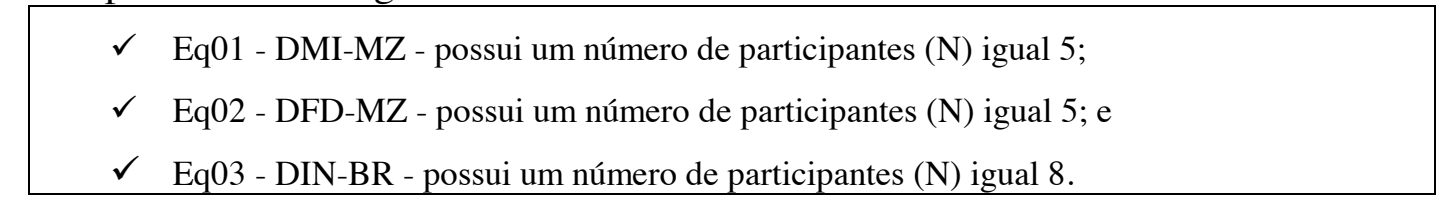

$\checkmark$ Nível de influência da estimativa de esforço por equipe é resultante do cálculo da somatória dos valores caracterizado pelos participantes no término da configuração dos fatores para a métrica Esforco.part associada para compor a estratégia. A fórmula de cálculo do nível de influência de cada equipe é descrita abaixo:

\begin{tabular}{|l|l|l|}
\hline Eq01.nie1 $=\frac{\sum(\text { nie } 1)}{N}=\frac{18}{5}=3,6$ & Eq02.nie $2=\frac{\sum(\text { nie } 2)}{N}=\frac{18}{5}=3,6 \quad$ Eq03.nie $3=\frac{\sum(\text { nie } 3)}{N}=\frac{29}{8}=3,63$ \\
\hline
\end{tabular}

Onde nie1 - é o nível de influência a estimativa de esforço associado ao participante.

$\checkmark$ Estimativa de esforço por equipe é resultante do cálculo da somatória dos valores atribuídos aos fatores de complexidade técnica, ambientais para alimentar a métrica Esforco dividida pelo número de participantes. A fórmula de cálculo da estimativa do esforço despendido de cada equipe é descrita abaixo:

$$
\begin{array}{|l|l|l|}
\hline \text { Eq01.ed1 }=\frac{\sum(e d 1)}{N}=\frac{3,42}{5}=0,68 & \text { Eq02.ed } 2=\frac{\sum(e d 2)}{N}=\frac{3,59}{5}=0,72 \quad \text { Eq03.ed } 3=\frac{\sum(e d 3)}{N}=\frac{5,59}{8}=0,74 \\
\hline
\end{array}
$$

Onde ed1 - é a estimativa de esforço despendido por equipe.

$\checkmark$ Distancia media geográfica e temporal das equipes. A configuração da distancia geográfica e temporal esta representada por três lugares diferentes, Moçambique, Brasil e Portugal. Neste ultimo encontra-se o gerente global responsável por distribuir os artefatos para configuração dos estudos experimentais. A fórmula de cálculo do índice médio da distancia geográfica ( SDI ) e distancia espacial (TDI) e descrita abaixo:

$$
S D I=\frac{(5353 \times 10 \times 1) \times(5711 \times 10 \times 8) \times(4080 \times 8 \times 1)}{\left(18^{2}-18\right) / 2}=\frac{543050}{153}=3,55
$$


Onde $S D I$ - índice media da distancia geográfica entre as equipes dispersas a nível global.

$$
T D I=\frac{(5 \times 10 \times 8) \times(1 \times 10 \times 1) \times(4 \times 1 \times 8)}{\left(18^{2}-18\right) / 2}=\frac{422}{153}=2,89
$$

Onde $T D I$ - índice media da distancia temporal entre as equipes dispersas a nível global.

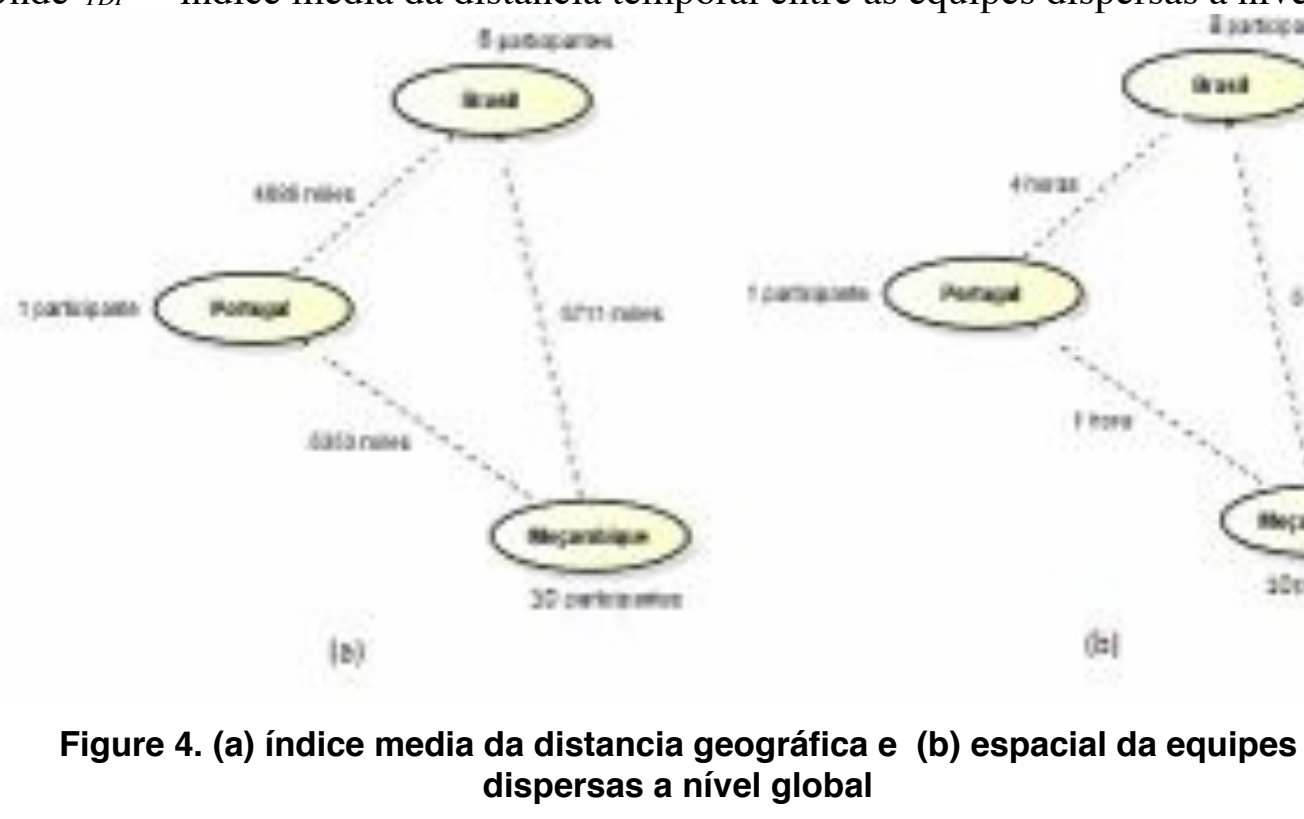

\subsection{Interpretação dos resultados obtidos}

Com base na análise da fundamentação dos elementos da estratégia é calculada a função objetiva, índice para alocação da EReq em DDS, que consiste em multiplicar todos os elementos que representam a estratégia segundo a equação (Eq6).

$$
\rho(\mathrm{DI})_{E q}=\rho(\operatorname{Corr} 1) \times \text { nie } \times e d \times S D I \times T D I
$$

Onde: $\rho(\mathrm{DI})_{E q}$ - é o índice da alocação da EReq em DDS, $\rho($ Corr1) - é a correlação da métricas (indicador) para alocação da EReq. nie - é o nível de influência de estimativa de esforço por equipe, ed - é a estimativa de esforço despendido por equipe, SDI - é o índice da distancia geográfica (espacial) e e TDI- é o índice da distancia temporal. Portanto, a geração do índice da alocação $\rho\left(\right.$ DI ${ }_{E q}$ foi realizada com base nos casos de uso configurada por cada equipe. Assim, o objetivo foi dar prioridade para equipe que obtiver o menor índice de alocação, visto que quanto menor for ed maior é a compreensão dos casos de uso. Os dados contendo os valores do índice da alocação $\rho(\text { DI })_{E q}$ são descritos para o cálculo de cada equipe abaixo:

$$
\begin{aligned}
& \rho(\mathrm{DI})_{E q 01}=0,62 \times 3,6 \times 0,68 \times 3,55 \times 2,89=15,57 \\
& \rho(\mathrm{DI})_{E q 02}=0,62 \times 3,6 \times 0,72 \times 3,55 \times 2,89=16,48 \\
& \rho(\mathrm{DI})_{E q 03}=0,62 \times 3,63 \times 0,74 \times 3,55 \times 2,89=17,09
\end{aligned}
$$


Estes resultados são projetados como $\rho(\mathrm{DI})_{E q}$, índice da alocação da EReq de DDS. A ordenação para classificar o ranking da equipe, apresentada na Figura 5, a melhor posicionada para ser selecionada para realizar a etapa é medida de acordo com o valor $\rho(\text { DI })_{E q}$. Se a medida dos valores das equipes tender a 0 (zero), maior é a chance de ser selecionada a EReq. Em análise vemos que os resultados dos índices da alocação são diferentes devido as variáveis ed, nie (o que significa que encontramos qualquer $\rho(\text { DI })_{E q}$ positiva ou negativa), atribuídas por cada equipe respectivamente. Como os valores $\rho($ Corrl), SDI e TDI são constantes eles não afetam a variância dos resultados. Portanto, os valores das variáveis tornam-se desequilibradas, podemos concluir que, quanto menor for a variável ed, maior é o entendimento da regra de negócio para especificar o requisito e menor é o valor atribuído pelo participante a variável nie .

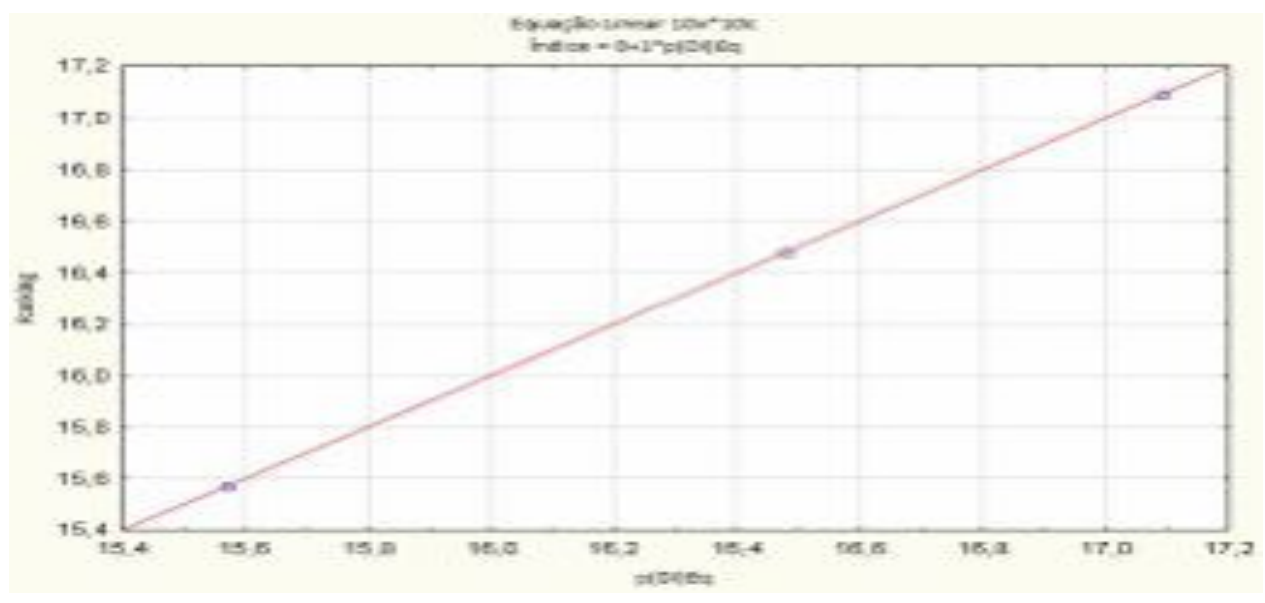

Figure 5. Ranking das equipes para alocação da EReq em DDS

Deste modo, pode-se argumentar que o índice de alocação é capaz de gerar resultados significativos na alocação da etapa a equipes dispersas em nível global para realizar a EReq e suas tarefas correspondentes. Assim, quanto menor for o $\rho(\mathrm{DI})_{E q}$, maior é a chance de a equipe ser selecionada. Nesse estudo a equipe ( $\left.\rho(\mathrm{DI})_{E q 01}\right)$ possui o menor valor do índice por que se aproxima do valor 15,4 pretendido.

\section{Conclusões}

Os resultados obtidos deste estudo apontam para as seguintes conclusões: (1) a validação empírica do estudo experimental da validação das métricas mostrou a importância de compreender a natureza dinâmica do DDS e (2) a correlação de Esforco e Esforco.part possui um valor limite de $(0,62)$, o que evidencia que tais métricas são fortemente correlacionadas para direcionar a alocação da Ereq a equipes dispersas a nível global no modelo de negocio offshore outsourcing .

A estratégia limita-se na validação baseado no diagrama de case de uso e UML, não considerando outras abordagens de desenvolvimento de software como ModelDriven Development (MDD) e orientado a aspectos. Outra limitação é a instrumentação dos gerentes locais no projeto piloto que poderá causar alguma ameaça a validação, visto que a amostra foi composta por estudantes, o que de certa forma, impede a generalização de resultados obtidos de tais experimentos. Como trabalho futuro 
pretende-se aplicar replicações do estudo experimental com tamanho de amostras maiores para que seja possível alcançar a normalidade dos valores observados de modo a obter evidencias reais de que as métricas podem ser utilizadas como indicadores de alocação da EReq em DDS e investigar a possibilidade de aplicar a estratégia em fábrica de software com o objetivo de generalizar os resultados obtidos sobre a viabilidade da uso da estratégia.

Agradecimentos: CNPq (Programa PEC-PG) pelo apoio financeiro.

\section{Referências}

Basili, V. and Rombach, H. (2009) The tame project: Towards improvement oriented software environments. In: IEEE Transactions on Software Engineering, 1988, p. 758-773.

Corder, G. and Foreman, D. Nonparametric statistics for non-statisticians: A step by step approach. USA:Wiley.

Damian, D. and Zowghi, D. (2002) The impact of stakeholders geographical distribution $\mathrm{o}$ requirements engineering in a multi-site development organization. In: International Conference on Requirements Engineering, p. 319-328.

Gotel, O., Kulkarni, V. and scharff, C. (2010) From student to software engineer in the indian it industry: A survey of training. In: Proceedings of the 23rd Conference on Software Engineering Education and Training (CSEET10), IEEE-CS Press: Silver Spring MD, p. 23-33.

Herbsleb, J. D. and Moitra, (2001) D. Global software developmentIEEE Software, p. 16-20.

Karner, G. (1993) Metrics for objectory. diploma thesis, university of linkoping. Sweden.

Fenton, N.E and Pfleeger, S. L. (1991) Software Metrics: A rigorous and practical approach, second edition, PWS Publishing Company.

Lamersdorf, A., Munch, J. and Rombach, D. (2009) A survey on the state of the practice in distributed software development: Criteria for task [9] allocation. In: IEEE Int. Conf. on Global Software Engineering, ICGSE, p. 41-50.

Matusse, E. A., Huzita, E. M., Tait, T. F. (2012) Métricas e indicadores para auxiliar na distribuição das etapas do processo em desenvolvimento distribuído de software: Uma revisão sistemática. In: XXXVIII Conferencia Latino-americana En Informática.

O'Leary, M. B. and Cummings, J. N. (200\&) The spatial, temporal, and configurational characteristics of geographic dispersion in teams. MIS Quarterly, p. 433-452, 2007.

Prikladnicki, R. And Audy, J. L. N. (2008) Desenvolvimento distribuído de software. Elsevier..

Ribu, V. Goal question metric paradigm. (2001) In: Encyclopdia of Software Engineering, p. 527-532.

Spearman, C. (1994) The proof and measurement of association between two things. In: American Journal of Psychology,15(1):72101.

Shapiro, S.S and Wilk, M.B. (1965) An Analysis of Variance Test for Normality (Complete Sample). Biometrika, V.3,n.52, p. 591-611. 